\title{
Determining association of rho kinase 1 gene polymorphisms with risk of Alzheimer's disease: a multicenter pilot study
}

\author{
Ying Gao ${ }^{1 \#}$, Xia Li ${ }^{2 \#}$, Xiao-Hong Liu ${ }^{3}$, Qian-Hua Zhao ${ }^{4}$, Xiang-Qian Che ${ }^{1}$, Qi-Hao Guo ${ }^{4}$, Ru-Jing Ren ${ }^{1}$, \\ Gang Wang ${ }^{1}$
}

${ }^{1}$ Department of Neurology \& Institute of Neurology, Ruijin Hospital, Shanghai Jiao Tong University School of Medicine, Shanghai 200025, China; ${ }^{2}$ Alzheimer's Disease and Related Disorders Center, Department of Geriatric Psychiatry, Shanghai Mental Health Center, Shanghai Jiao Tong University School of Medicine, Shanghai 200030, China; ${ }^{3}$ Department of Neurology, Shanghai Putuo District People's Hospital, Shanghai 200060, China; ${ }^{4}$ Department of Neurology \& Institute of Neurology, WHO Collaborating Center for Research and Training in Neurosciences, Huashan Hospital, Fudan University, Shanghai 200040, China

Contributions: (I) Conception and design: G Wang, RJ Ren; (II) Administrative support: None; (III) Provision of study materials or patients: None; (IV) Collection and assembly of data: All authors; (V) Data analysis and interpretation: All authors; (VI) Manuscript writing: All authors; (VII) Final approval of manuscript: All authors.

"These authors contributed equally to this work.

Correspondence to: Ru-Jing Ren; Gang Wang. Department of Neurology \& Institute of Neurology, Ruijin Hospital affiliated to Shanghai Jiao Tong University School of Medicine, 197 Ruijin 2nd Road, Shanghai 200025, China. Email: doctoren2001@126.com; wgneuron@hotmail.com.

\begin{abstract}
Background: In addition to the increasing evidence for a molecular mechanism of rho kinase 1 (ROCK1) in Alzheimer's disease (AD), there are several published studies regarding the relationship between ROCK1 gene polymorphisms and neurological diseases. However, it is unknown whether there is an association between the polymorphisms of ROCK1 and AD. We sought to identify the potential association between ROCK1 gene polymorphisms and AD in the Chinese Han population.

Methods: A total of 295 patients with AD and 206 healthy controls from multiple centers were enrolled in this study. Three single-nucleotide polymorphisms (SNPs) (rs35996865, rs11873284, and rs2127958) in ROCK1 gene were analyzed using Sanger sequencing.

Results: We did not find any significant differences between $\mathrm{AD}$ and control groups with regards to the frequency of these three ROCK1 polymorphisms. Further, the three SNP genotype frequencies and allele frequencies did not show significant differences between patients of $\mathrm{AD}$ and controls in APOE4-stratified subjects $(\mathrm{P}>0.01)$. Additionally, the three SNPs did not show significant differences even when adopting a four-inheritance model by logistic regression.

Conclusions: This is the first multicenter pilot study to evaluate the contribution of ROCK1 genetic variance to $\mathrm{AD}$ risk. Our data demonstrated that the ROCK1 gene may not influence the risk of $\mathrm{AD}$ by interacting with $A P O E$ among Chinese Han people.
\end{abstract}

Keywords: Alzheimer's disease (AD); rho-kinase 1; apolipoprotein E (APOE); polymorphism

Submitted May 15, 2018. Accepted for publication May 18, 2018.

doi: $10.21037 /$ atm.2018.05.51

View this article at: http://dx.doi.org/10.21037/atm.2018.05.51

\section{Introduction}

Alzheimer's disease (AD), the most common dementia, is a genetically complex disease which can be caused by genetic and environmental factors, individually and in interaction with each other. Mutations in three genes (APP, PSEN1, and PSEN2) have been identified to be associated with autosomal dominant forms of familial $\mathrm{AD}$ (1). As mutations that cause familial $\mathrm{AD}$, other heritable genetic risk factors also contribute to individual's susceptibilities to AD. For 
Table 1 Demographic detail of the sample set

\begin{tabular}{lcc}
\hline Characteristics of participants & Cases with AD & Controls \\
\hline Total sample & 295 & 206 \\
Male & 116 & 92 \\
Female & 179 & 114 \\
Age (years) & $70.5 \pm 10.3$ & $70.9 \pm 9.0$ \\
MMSE score & $14.7 \pm 7.2$ & $28.1 \pm 2.2$ \\
\hline
\end{tabular}

The ages and MMSE scores are means and standard deviations. $A D$, Alzheimer's disease; MMSE, mini-mental state examination.

example, Apolipoprotein E (APOE) $\varepsilon 4$ allelic variants have been identified (2), and found to be linked to a major increase in the risk for susceptibility to $\mathrm{AD}$ in different populations (3). Furthermore, genome-wide association studies (GWAS) have identified more than 20 genetic loci associated with the risk of $\mathrm{AD}$, including BIN1, PICALM, $C L U, C R 1, M S 4 A 6 A$ and others (4).

Rho kinases (ROCKs) are serine/threonine kinases first identified as downstream effector of Rho GTPase. ROCK genes consist of two paralogs: ROCK1 and ROCK2, sharing $65 \%$ homology in amino acid sequences and $92 \%$ similarity in kinase domains (5). ROCKs constitute an important intracellular signaling system that participates in the regulation of a multitude of cellular functions, such as proliferation, differentiation, metabolism and apoptosis $(6,7)$. Notably, several studies have suggested that ROCK kinases can induce the processing of APP into the toxic $\beta$-amyloid (A $\beta$ ) 1-42 peptide and furthermore, inhibitors of ROCKs, such as statins and NSAIDs, can inhibit this toxic APP processing $(8,9)$. ROCK1 is located on chromosome 18 (18q11.1) and is expressed mainly in hypothalamus and hippocampus. Recent research has found that ROCK1 is elevated in mild cognitive impairment (MCI) and $\mathrm{AD}$ brain, and knockout of the ROCK1 gene reduced the production of $A \beta$ (10). Similarly, our recent study suggested that ROCK1 increases $\mathrm{A} \beta$ clearance by modulating autophagosome formation and is also involved in tau hyperphosphorylation and cytoskeleton disruption by miRNA-146a (11-13). These discoveries elucidated that ROCK1 is involved in modulating metabolism of both $\mathrm{A} \beta$ and tau, the constituents of hallmark $\mathrm{AD}$ pathologies.

Interestingly, there are several published studies in regard to the relationship between ROCK1 gene polymorphisms and diseases that threaten the health of people (14-18)
With increasing evidence for a molecular mechanism of ROCK1 in AD pathogenesis, ROCK1 genetic variance as a potential contributor to $\mathrm{AD}$ risk is still not completely understood. Here, we put forward a hypothesis that ROCK1 gene polymorphisms affect potential risk for $\mathrm{AD}$, and we test this with a case-control study $(\mathrm{n}=501)$ to determine the prevalence of the common single-nucleotide polymorphisms (SNPs) of ROCK1 (rs35996865, rs11873284, and rs2127958) among healthy and AD patients in the Chinese Han population, and investigate the association between these polymorphisms and $\mathrm{AD}$ risk.

\section{Methods}

\section{Study population}

A total of $295 \mathrm{AD}$ patients of Chinese Han ethnicity (179 women and 116 men; $70.5 \pm 10.3$ years old were recruited; the Mini-Mental State Examination (MMSE) score mean for these patients was $14.7 \pm 7.2$ ) were enrolled from the outpatient Clinic at the Department of Neurology, Ruijin Hospital affiliated to Shanghai Jiao Tong University School of Medicine, and at the Department of Geriatric Psychiatry, Shanghai Mental Health Center, Shanghai Jiao Tong University School of Medicine, and Department of Neurology \& Institute of Neurology, Huashan Hospital, Fudan University. All subjects received a detailed neurological examination and underwent a psychiatric interview. The patients had a clinical diagnosis of probable dementia of Alzheimer type according to NINCDS-ADRDA (National Institute of Neurological and Communicative Disorders and Stroke-Alzheimer's Disease and Related Disorders Association). Two hundred and six gender, age, and ethnic background-matched nondemented elderly controls (NEC) (114 women and 92 men; $70.9 \pm 9.0$ years old at the recruitment; average MMSE score, $28.1 \pm 2.2$ ) were also recruited. The makeup of male and female subjects did not vary significantly $(\mathrm{P}>0.05)$ (Table 1). The NEC subjects were free of neurological or psychiatric disorders by medical history, physical examinations, laboratory examinations, and with a MMSE score over 26 (Table 1). All AD patients and NEC were unrelated Chinese Han. Informed consent for participation in the study was obtained either directly, or from a guardian of each patient. This study was approved by the Research Ethics Committee, Ruijin Hospital affiliated to Shanghai Jiao Tong University School of Medicine, Shanghai, China. 


\section{SNP selection}

The preliminary screening criteria for $R O C K 1$ gene polymorphisms were (I) minor allele frequency $>5 \%$ in the Chinese Han population; (II) on the basis of previous literature data. This resulted in selection of three SNPs in ROCK1 (rs35996865, rs2127958, rs11873284) for inclusion in this study.

\section{Blood samples, DNA isolation and genotyping}

Genomic DNA was isolated from peripheral blood through standardized phenol/chloroform extraction. Three single nucleotide polymorphisms (rs35996865, rs2127958, rs11873284) and APOE were genotyped through PCR using flanking primers and Sanger sequencing. Primers of ROCK1 were designed according to the RefSeq gene sequence. Optimized primer sequences are listed in supplementary Table S1. Primers of APOE were used as described in Zivelin et al. (19). PCR conditions are available on request.

\section{Statistical analysis}

Results are expressed as mean $\pm \mathrm{SD}$ or percentage as indicated. To compare the differences of mean values between two groups, unpaired Student's $t$-test was used. Goodness-of-fit to the Hardy-Weinberg equilibrium (HWE) and differences in genotype and allele frequencies between the cases and controls were calculated by Chisquared analysis with Fisher's exact tests. The criterion for significance level was set at a $\mathrm{P}$ value of 0.01 for all tests. All probability values were based on two-tailed tests. SPSS was used for statistical analysis. Haplotype analysis was performed by using online software, SHEsis (http:// analysis.bio-x.cn/myAnalysis.php), SNPStats (https://www. snpstats.net/snpstats/start.htm?q=snpstats/start.htm) was used to evaluate the association between SNPs and the risk of $\mathrm{AD}$ under 4 inheritance models, including codominant, overdominant, dominant, and recessive models.

\section{Results}

In the present study, a total of 295 patients with $\mathrm{AD}$ and 206 unrelated gender- and age-matched $(\mathrm{P}>0.05)$ controls were investigated. Table 1 shows the clinical features of the study population.

Table 2 shows the distribution of genotypes and alleles for ROCK1 and APOE\&4 between the case and control. No significant deviations from HWE were found for ROCK1 in two groups. Our study demonstrated no significant differences between groups among three ROCK1 polymorphisms in allele frequencies and genotype distributions (Table 2). Further, the three SNP genotype frequencies and allele frequencies also did not show significant differences between patients of $\mathrm{AD}$ and controls in APOE\&4-stratified subjects (Table 3) $(\mathrm{P}>0.01)$.

To further investigate the ROCK1 genetic association of $\mathrm{AD}$, four-inheritance models were assumed and analysis was performed by logistic regression among 84 -carriers. The three SNPs did not show significant differences adopting

Table 2 Genotype and allele frequencies for ROCK1 and APOE

\begin{tabular}{|c|c|c|c|c|c|c|}
\hline \multirow{2}{*}{ Gene } & \multirow{2}{*}{ Genotype/alleles } & \multicolumn{2}{|c|}{ Cases with $A D$} & \multicolumn{2}{|c|}{ Controls } & \multirow{2}{*}{$\mathrm{P}$} \\
\hline & & $\mathrm{n}$ & Total $^{a}$ & $\mathrm{n}$ & Total $^{\mathrm{a}}$ & \\
\hline ROCK1 & TT/TG/GG & $217 / 62 / 4$ & 283 & $168 / 36 / 1$ & 205 & 0.282 \\
\hline rs35996865 & $\mathrm{T} / \mathrm{G}$ & $496 / 70$ & & $372 / 38$ & & 0.128 \\
\hline ROCK1 & $\mathrm{TT} / \mathrm{TC} / \mathrm{CC}$ & $90 / 138 / 59$ & 287 & $62 / 107 / 35$ & 204 & 0.546 \\
\hline ROCK1 & $\mathrm{AA} / \mathrm{AG} / \mathrm{GG}$ & $219 / 58 / 4$ & 281 & $172 / 33 / 1$ & 206 & 0.242 \\
\hline rs11873284 & $\mathrm{A} / \mathrm{G}$ & $496 / 66$ & & $377 / 35$ & & 0.100 \\
\hline$A P O E$ & $(-,-) /(-,+) /(+,+)$ & $164 / 104 / 27$ & 295 & $169 / 35 / 2$ & 206 & $<0.0001$ \\
\hline$\varepsilon 4$ & $-/+$ & $432 / 158$ & & $373 / 39$ & & $<0.0001$ \\
\hline
\end{tabular}

$\mathrm{P}, \mathrm{P}$ value (Chi square test or Fisher exact test); $\mathrm{n}$, number; ${ }^{\text {a }}$, numbers do not always add up to total numbers because of missing values in the sanger sequencing. AD, Alzheimer's disease. 
Table 3 Association of ROCK1 SNPs with AD in APOE4 stratified samples

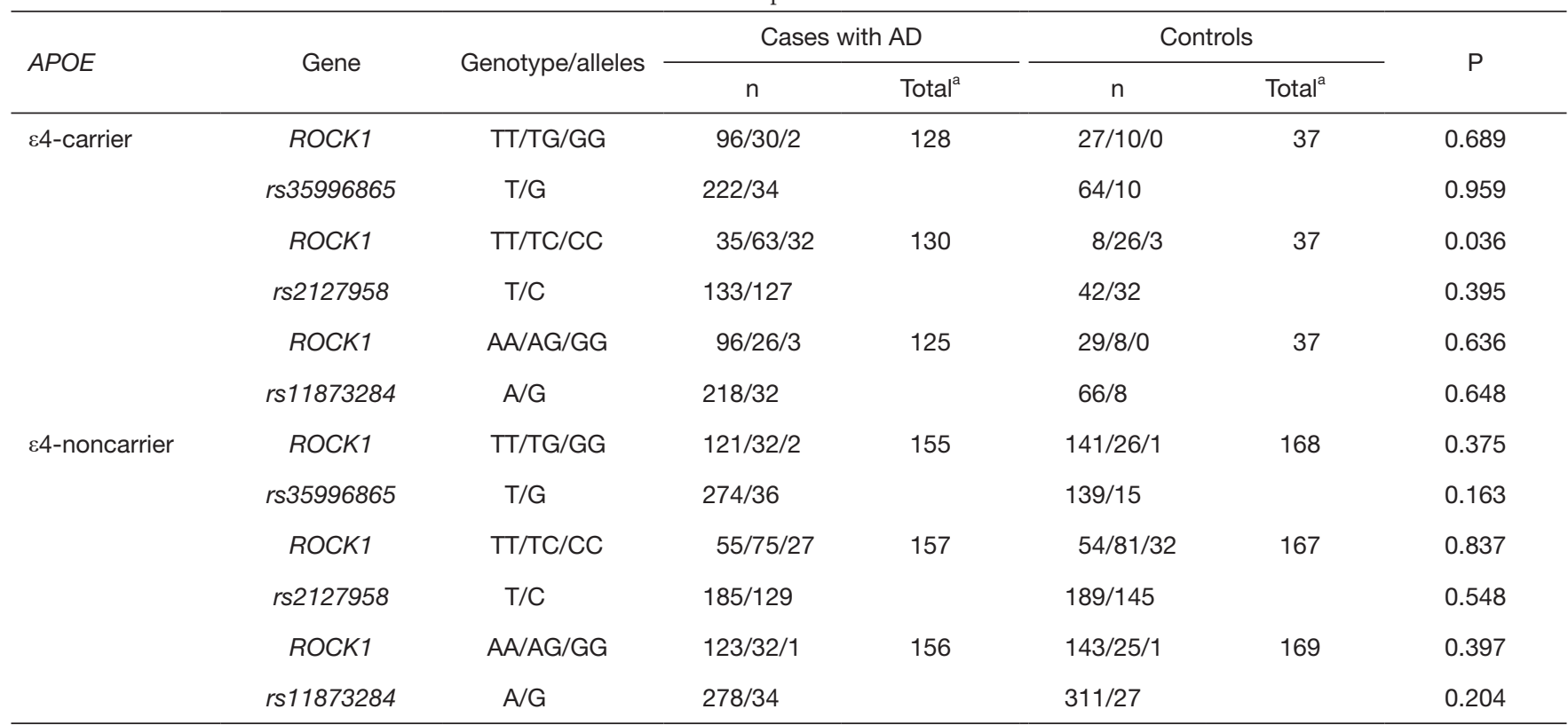

$\mathrm{P}, \mathrm{P}$ value (Chi square test or Fisher exact test); $\mathrm{n}$, number; ${ }^{\mathrm{a}}$, numbers do not always add up to total numbers because of missing values in the sanger sequencing. $\varepsilon 4$-carrier, carriers of at least one 4 allele of the Apolipoprotein $E$ gene; $\varepsilon 4$-noncarrier, subjects not carrying an $\varepsilon 4$ allele of the Apolipoprotein E gene. AD, Alzheimer's disease; SNPs, single-nucleotide polymorphisms

any of the four-inheritance model (Table 4).

\section{Discussion}

To the best of our knowledge, this is the first study to investigate the contribution of ROCK1 gene variants to risk for $\mathrm{AD}$. According to molecular neuropathogenic investigations distinct from GWAS, we put forward a novel hypothesis that ROCK1 variation could account for some risk of developing $\mathrm{AD}$. Unfortunately, our outcomes do not show evidence of ROCK1 associations to AD risk among AD patients, even when stratified by $A P O E$ genotype. As the strongest genetic determinant of $\mathrm{AD}$, APOE\&4 allele associates with increased incidence of $\mathrm{AD}$, whereas the $\varepsilon 2$ allele decreases risk. Studies in humans and transgenic mice showed that APOE regulates A $\beta$ metabolism, aggregation and clearance in the brain in an isoform-dependent manner (20-23). For example, APOE $\varepsilon 4$ astrocytes eliminate $A \beta$ plaques less effectively than APOE $\varepsilon 3$ astrocytes (24), and clearance of $A \beta$ by endocytosis is impaired by APOE\&4 (25). Interestingly, Iizuka and coworkers found ROCK1 immunoreactivity was distributed in astrocytes and Bergmann glial cells that actually produce APOE in the CNS (26). Again, our previous study shows that ROCK1 colocalizes with highly fibrilized $\mathrm{A} \beta$ and that activated ROCK1 inhibits the autophagic clearance of A $\beta$ (12). Both APOE and ROCK1 colocalize with plaque-associated amyloid and glial cells, suggesting a role for ROCK1 in pathogenesis of $\mathrm{AD}$ by interacting with APOE4. Follow-up functional studies are still needed to confirm these findings.

Interestingly, two studies have identified ROCK1 as a risk factor of brain diseases. Robert and colleagues investigated the role of $R O C K 1$ gene variations played in ischemic stroke and found that there were obvious associations between ROCK1 gene variants (rs2127958, rs11873284) and ischemic stroke in Caucasian women (14). Evidence from clinical studies has shown that ROCK activity was elevated in patients with acute ischemic stroke (27) and inhibition of ROCK activity by statins probably helps to prevent ischemic stroke (28-30). Non-synonymous SNPs (rs111874856, rs112130712, rs112108028, rs73963110) are involved in the risk of Behcet's disease in the Turkish population (15). The frequencies of these four variants are extremely rare to absent in Chinese populations according to the NCBI database. Both studies looked at Caucasians, which have a different genetic background from Asians. Additionally, 
Table 4 Four genetic modes of inheritance for the three studied polymorphisms in 84 -carrier (adjusted by gender and age)

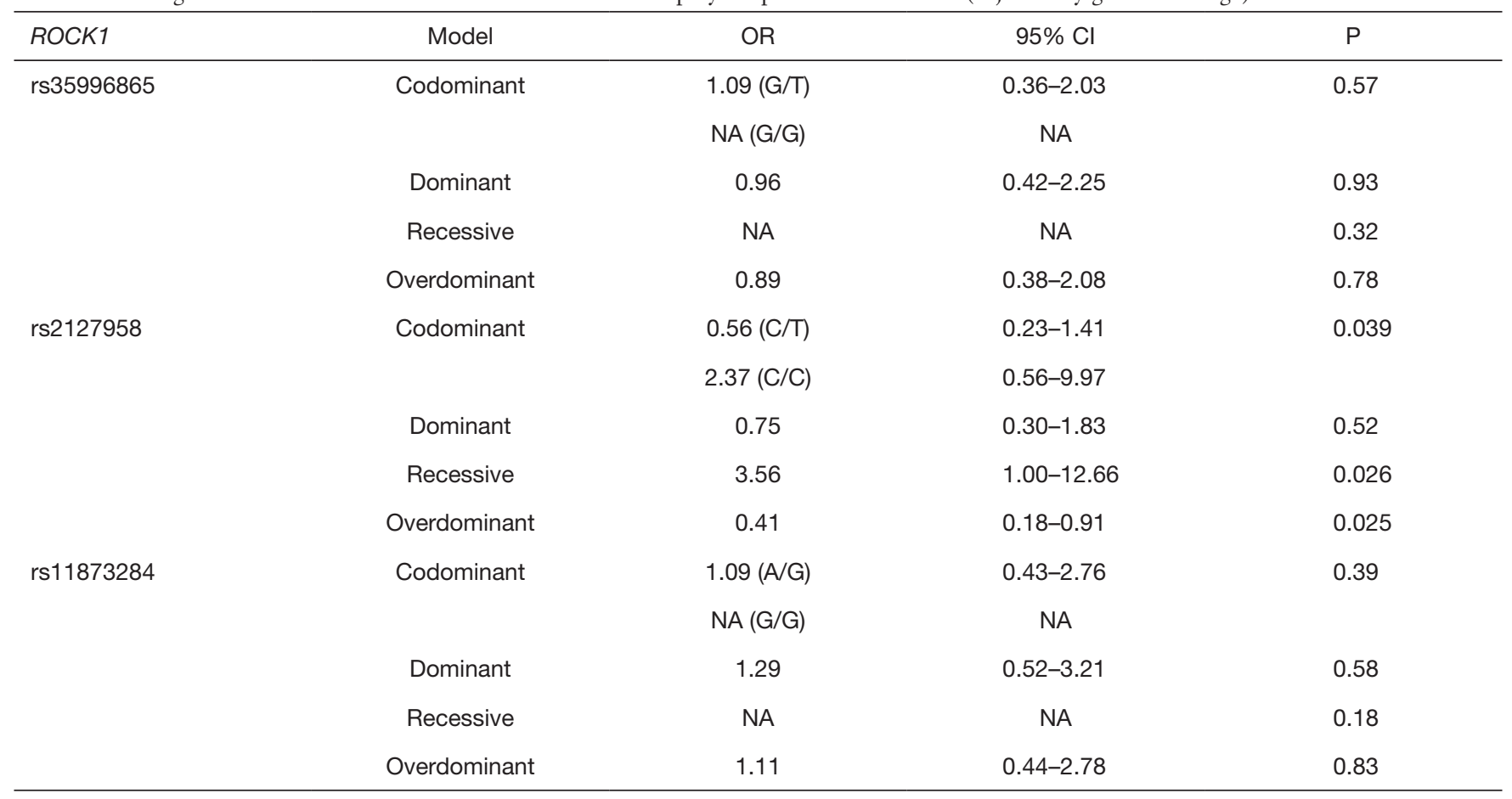

$\mathrm{P}, \mathrm{P}$ value; OR, odds ratio; $\mathrm{Cl}$, confidence interval; NA, not applicable; codominant, the contributions of both alleles are visible in the phenotype; Dominant, the effect of one allele is visible when being homozygotes or heterozygotes; Recessive, the effect of one allele is visible only when being homozygotes; Overdominant, the phenotype of the heterozygote lies outside the phenotypical range of both homozygous parents.

the ROCK1 gene polymorphism rs35996865 mapping to the 5'-UTR was significantly associated with colorectal cancer (16), obesity-related metabolic syndrome (17), renal cell carcinoma (18) and respiratory distress syndrome (31), but not AD. Nonetheless, we have detected elevated ROCK1 activity in the peripheral blood of AD patients (unpublished data) and found that ROCK1 plays an important role in pathogenic mechanisms of AD. However, our association study did not show that any of the three SNPs examined increases the risk of AD.

Results from this study may have some implications for future work, in order to clarify the role for increasing $\mathrm{AD}$ risk of the APOE\&4 allele in association with ROCK1 gene variants. The transcription level of the $A P O E$ gene should be considered with ROCK1 SNP status to determine their potential cooperative role in the progression of $\mathrm{AD}$. Further, the combined effects of APOE4 and ROCK1 on senile plaque clearance and phagocytosis by astrocytes still remains to be examined. One of the limitations of this study is small sample size. Larger sample size and different ethnic groups would be helpful for explaining the involvement of
ROCK1 in AD pathogenesis. Moreover, further analysis of the correlations of other polymorphisms in the ROCK1 gene and clinical manifestations is warranted in future.

In summary, we present here the first multicenter pilot study to evaluate the contribution of ROCK1 variants to $\mathrm{AD}$ risk. Our data demonstrated that the ROCK1 gene may not influence the risk of $\mathrm{AD}$ by interacting with $A P O E$ among Chinese Han people.

\section{Acknowledgements}

Funding: We thank the patients and their families for their participation in this project. We thank Dr. Eric B. Dammer at the Department of Biochemistry, Center for Neurodegenerative Diseases, Emory University School of Medicine for his critical reading. This study was supported by the National Natural Science Foundation of China (No. 81671043) and the "Shuguang Program (16SG15)" supported by Shanghai Education Development Foundation and Shanghai Municipal Education Commission. The authors declare no competing financial interests. 


\section{Footnote}

Conflicts of Interest: The authors have no conflicts of interest to declare.

Ethical Statement: This study was approved by the Research Ethics Committee, Ruijin Hospital affiliated to Shanghai Jiao Tong University School of Medicine, Shanghai, China (No. 2016-127). Informed consent for participation in the study was obtained either directly, or from a guardian of each patient.

\section{References}

1. Loy CT, Schofield PR, Turner AM, et al. Genetics of dementia. Lancet 2014;383:828-40.

2. Borgaonkar DS, Schmidt LC, Martin SE, et al. Linkage of late-onset Alzheimer's disease with apolipoprotein E type 4 on chromosome 19. Lancet 1993;342:625.

3. Corder EH, Saunders AM, Strittmatter WJ, et al. Gene dose of apolipoprotein E type 4 allele and the risk of Alzheimer's disease in late onset families. Science 1993;261:921-3.

4. Scheltens P, Blennow K, Breteler MM, et al. Alzheimer's disease. Lancet 2016;388:505-17.

5. Nakagawa O, Fujisawa K, Ishizaki T, et al. ROCK-I and ROCK-II, two isoforms of Rho-associated coiled-coil forming protein serine/threonine kinase in mice. FEBS Lett 1996;392:189-93.

6. Riento K, Ridley AJ. Rocks: multifunctional kinases in cell behaviour. Nat Rev Mol Cell Biol 2003;4:446-56.

7. Hensel N, Rademacher S, Claus P. Chatting with the neighbors: crosstalk between Rho-kinase (ROCK) and other signaling pathways for treatment of neurological disorders. Front Neurosci 2015;9:198.

8. Zhou Y, Su Y, Li B, et al. Nonsteroidal anti-inflammatory drugs can lower amyloidogenic Abeta 42 by inhibiting Rho. Science 2003;302:1215-7.

9. Tang BL, Liou YC. Novel modulators of amyloidbeta precursor protein processing. J Neurochem 2007; 100:314-23.

10. Henderson BW, Gentry EG, Rush T, et al. Rho-associated protein kinase 1 (ROCK1) is increased in Alzheimer's disease and ROCK1 depletion reduces amyloid-beta levels in brain. J Neurochem 2016;138:525-31.

11. Hu YB, Dammer EB, Ren RJ, et al. The endosomallysosomal system: from acidification and cargo sorting to neurodegeneration. Transl Neurodegener 2015;4:18.
12. Hu YB, Zou Y, Huang Y, et al. ROCK1 Is Associated with Alzheimer's Disease-Specific Plaques, as well as Enhances Autophagosome Formation But not Autophagic Abeta Clearance. Front Cell Neurosci 2016;10:253.

13. Wang G, Huang Y, Wang LL, et al. MicroRNA-146a suppresses ROCK1 allowing hyperphosphorylation of tau in Alzheimer's disease. Sci Rep 2016;6:26697.

14. Zee RY, Wang QM, Chasman DI, et al. Gene variations of ROCKs and risk of ischaemic stroke: the Women's Genome Health Study. Clin Sci (Lond) 2014;126:829-35.

15. Oguz E, Demiryurek AT, Pehlivan Y, et al. Association of Rho-kinase 1 (ROCK1) gene polymorphisms with Behcet's disease. Mol Diagn Ther 2014;18:419-26.

16. Sari I, Berberoglu B, Ozkara E, et al. Role of rho-kinase gene polymorphisms and protein expressions in colorectal cancer development. Pathobiology 2013;80:138-45.

17. Tabur S, Oztuzcu S, Oguz E, et al. Association of Rho/ Rho-kinase gene polymorphisms and expressions with obesity-related metabolic syndrome. Eur Rev Med Pharmacol Sci 2015;19:1680-8.

18. Zhao R, Liu K, Huang Z, et al. Genetic Variants in Caveolin-1 and RhoA/ROCK1 Are Associated with Clear Cell Renal Cell Carcinoma Risk in a Chinese Population. PLoS One 2015;10:e0128771.

19. Zivelin A, Rosenberg N, Peretz H, et al. Improved method for genotyping apolipoprotein E polymorphisms by a PCR-based assay simultaneously utilizing two distinct restriction enzymes. Clin Chem 1997;43:1657-9.

20. Deane R, Sagare A, Hamm K, et al. apoE isoform-specific disruption of amyloid beta peptide clearance from mouse brain. J Clin Invest 2008;118:4002-13.

21. Jiang Q, Lee CY, Mandrekar S, et al. ApoE promotes the proteolytic degradation of Abeta. Neuron 2008;58:681-93.

22. Kok E, Haikonen S, Luoto T, et al. Apolipoprotein E-dependent accumulation of Alzheimer disease-related lesions begins in middle age. Ann Neurol 2009;65:650-7.

23. Schmechel DE, Saunders AM, Strittmatter WJ, et al. Increased amyloid beta-peptide deposition in cerebral cortex as a consequence of apolipoprotein $\mathrm{E}$ genotype in late-onset Alzheimer disease. Proc Natl Acad Sci U S A 1993;90:9649-53.

24. Simonovitch S, Schmukler E, Bespalko A, et al. Impaired Autophagy in APOE4 Astrocytes. J Alzheimers Dis 2016;51:915-27.

25. Li J, Kanekiyo T, Shinohara M, et al. Differential regulation of amyloid- $\beta$ endocytic trafficking and lysosomal degradation by apolipoprotein $\mathrm{E}$ isoforms. Journal of Biological Chemistry 2012;287:44593. 
26. Iizuka M, Kimura K, Wang S, et al. Distinct distribution and localization of Rho-kinase in mouse epithelial, muscle and neural tissues. Cell Struct Funct 2012;37:155-75.

27. Feske SK, Sorond FA, Henderson GV, et al. Increased leukocyte ROCK activity in patients after acute ischemic stroke. Brain Res 2009;1257:89-93.

28. Rawlings R, Nohria A, Liu PY, et al. Comparison of effects of rosuvastatin $(10 \mathrm{mg})$ versus atorvastatin $(40 \mathrm{mg})$ on rho kinase activity in caucasian men with a previous atherosclerotic event. Am J Cardiol 2009;103:437-41.

29. Liu PY, Liu YW, Lin LJ, et al. Evidence for statin

Cite this article as: Gao Y, Li X, Liu XH, Zhao QH, Che XQ, Guo QH, Ren RJ, Wang G. Determining association of rho kinase 1 gene polymorphisms with risk of Alzheimer's disease: a multicenter pilot study. Ann Transl Med 2018;6(22):434. doi: 10.21037/atm.2018.05.51 pleiotropy in humans: differential effects of statins and ezetimibe on rho-associated coiled-coil containing protein kinase activity, endothelial function, and inflammation. Circulation 2009;119:131-8.

30. Nohria A, Prsic A, Liu PY, et al. Statins inhibit Rho kinase activity in patients with atherosclerosis. Atherosclerosis 2009;205:517-21.

31. Kaya G, Sivasli E, Oztuzcu S, et al. Association of Rhokinase Gene Polymorphisms with Respiratory Distress Syndrome in Preterm Neonates. Pediatr Neonatol 2017;58:36-42. 
Supplementary

Table S1 Optimized primer sequences for rs35996865, rs11873284 and rs2127958

\begin{tabular}{ll}
\hline SNP & Primer sequences \\
\hline rs35996865-fw & tct gtt tcc tct ggg ttg ca \\
rs35996865-rv & tca cct ttc ctc aca cca ca \\
rs11873284-fw & cca cct tag cct ccc aaa gt \\
rs11873284-rv & gcc ttc ttt tgg act gtt tgg \\
rs2127958-fw & tgg cac tag gaa agg aca gtt \\
rs2127958-rv & agc atc tgg cct aag gga ct \\
\hline
\end{tabular}

SNP, single-nucleotide polymorphism; fw, forward strand; rv, reverse strand. 